\title{
Optimizing Movement of Students from Hostels to Lecture Rooms in a Potential World Class University Using Transportation Model
}

\author{
Michael C. Agarana1,2, Aniekeme I. Udoh ${ }^{3}$, Amenawon I. Ehigbochie ${ }^{3}$ \\ ${ }^{1}$ Department of Mathematics, Covenant University, Ota, Nigeria \\ ${ }^{2}$ Department of Mechanical Engineering Science, University of Johannesburg, Johannesburg, South Africa \\ ${ }^{3}$ Department of Computer Science and Management Information Systems, Covenant University, Ota, Nigeria \\ Email: michael.agarana@covenantuniversity.edu.ng
}

How to cite this paper: Agarana, M.C., Udoh, A.I. and Ehigbochie, A.I. (2018) Optimizing Movement of Students from Hostels to Lecture Rooms in a Potential World Class University Using Transportation Model. Applied Mathematics, 9, 633-646. https://doi.org/10.4236/am.2018.96044

Received: February 13, 2018

Accepted: June 16, 2018

Published: June 19, 2018

Copyright $\odot 2018$ by authors and Scientific Research Publishing Inc. This work is licensed under the Creative Commons Attribution International License (CC BY 4.0).

http://creativecommons.org/licenses/by/4.0/

\begin{abstract}
Transportation issue is one of the significant zones of utilization of Linear Programming Model. In this paper, transportation model is utilized to decide an ideal answer for the transportation issue in a run of the mill world class university utilizing Covenant University as a contextual analysis. Covenant University is a potential world class University. The quick development of Covenant University Campus over the most recent fourteen years affects its transportation framework. This paper particularly takes a gander at streamlining the time spent by the students moving from their lodgings to lecture rooms. Google guide was utilized to figure the separation and time between every cause and every goal. North-west corner technique, Least Cost strategy and Vogel's estimation technique were utilized to decide the underlying fundamental plausible arrangement (initial feasible solution) and MODI strategy was utilized to locate the ideal arrangement (optimal solution). The last outcome demonstrates that the development of understudies from hostel to lecture rooms can be streamlined if the total time spent is decreased.
\end{abstract}

\section{Keywords}

Transportation Model, World Class University, Optimization

\section{Introduction}

Transportation issue is an extraordinary class of linear programming issue and it is viewed as imperatively as a vital angle that has been considered in an extensive variety of operations including research areas. All things considered, it has been 
utilized as a part of recreation of a few genuine issues. In this way, streamlining transportation issue of factors has amazingly been critical to different controls [1]. Transportation issues are being confronted in urban regions because of increment in human exercises and most world class universities resemble little urban areas in light of their size and structures [2]. Additionally, today's grounds have moved toward becoming as mind boggling as urban zones, in view of their interesting attributes and between related impacts, most principle university's campuses are arranged as little urban communities/groups [2] [3]. Covenant University is a potential world class university and accordingly is considered as a little city. The university has been chosen as one of the best universities in West Africa. The university is located in Ota, Ogun State, Nigeria. As a potential world class university with its refined frameworks, all around prepared labs, all around prepared and qualified teachers, skilled students and an examination driven establishment the university flourishes for more brilliance [4] [5]. Aside the exceptionally normal or understood criteria of turning into a world class university like the one specified over, the transportation organized inside the campus is likewise vital to make campus life favorable and transportation less demanding for their students. In this paper time limiting transportation issue will be considered as against the standard cost limiting transportation issue [5] [6]. The essential contrast between the two is that the cost of transportation changes with varieties in the amount yet the time included stays unaltered and independent of the amounts [6]. Time limiting transportation model will be utilized to decide how the aggregate time spent by students making progress toward move from their hostels to their distinctive lecture rooms can be limited or enhanced to lessen exposure to un-conducive climate conditions and different variables [7] [8] [9]. The outcome that will be gotten from this work can be utilized by the University to deal with the transportation organized inside the campus. Arranging real university campus has been a genuine test as of late [10] [11]. Feasible transportation approaches have turned into a fundamental device to manage road transportation issues in most world class universities [11]. The call for manageable versatility is picking up fame worldwide for little and extensive groups including real university campus [11] [12]. Transportation issue is an extraordinary sort of linear programming issue where the goal is to limit the cost of dispersing an item from various sources or birthplaces to various destinations [13] [14] [15]. Transportation Problem (TP) has broad pragmatic application esteems [16] [17] [18]. In this manner, TP is a subject deserving of further research, in spite of the fact that it has been considered from various points of view by various researchers [19] [20] [21] [22]. The first formulation and discussion of a planar transportation model was introduced by Hitchcock (1941). Likewise, various scientists have done reviews on time transportation issue, for example, Sharma and Swarup (1977). As a general rule, because of changes in free market activity, climate conditions, street conditions and other instability variables, vulnerability transportation issue is especially essential [23] [24] [25]. 


\section{Problem Formulation}

Covenant University is used in this paper as a case study. The aim is to reduce the transportation time of students' movement from hostels to their respective lecture rooms. The Hostels represent the origins while the lecture rooms represent the destinations.

\subsection{Assumptions}

The following assumptions were made in this paper:

1) The time spent to move from one origin to a destination is always the same.

2) All the routes mentioned in this paper are always accessible.

3) Movement of students is always from the named origins to the named destinations.

4) Each student at the origin (hostel) visits each destination (lecture rooms).

5) Students attend lectures in each lecture room at least once a week (that is, from origin (hostel) to destination (lecture rooms)).

\subsection{Transportation Tableau}

In this paper ten origins representing the hostels and eight destinations representing the lecture rooms were considered.

Table 1 shows the different origins and destinations while Table 2 shows the transportation problem tableau. The distance between the origins and destinations are shown in the cells. The total time and distance covered in each route was determined using goggle map. The mathematical modeling of this transportation problem is a special linear programming problem in which the objective function is to minimize the time of transportation subject to the demand and supply constraints. The values in each cell represents the time per unit distance between one origin and one destination. They are measured in (meter $\times 100)$. Along the outer rim in the tableau is the actual distance and expected distance

Table 1. Origins and destinations considered.

\begin{tabular}{|c|c|c|c|}
\hline \multicolumn{2}{|c|}{ Origin } & \multicolumn{2}{|l|}{ Destination } \\
\hline Esther Hall & $\mathrm{O}_{1}$ & College of Science and Technology & $\mathrm{D}_{1}$ \\
\hline Mary Hall & $\mathrm{O}_{2}$ & College of Developmental Studies & $\mathrm{D}_{2}$ \\
\hline Deborah Hall & $\mathrm{O}_{3}$ & Lecture Theatre One & $\mathrm{D}_{3}$ \\
\hline Lydia Hall & $\mathrm{O}_{4}$ & Lecture theatre two & $\mathrm{D}_{4}$ \\
\hline Dorcas Hall & $\mathrm{O}_{5}$ & Chemical and Petroleum Engineering Block & $\mathrm{D}_{5}$ \\
\hline Peter Hall & $\mathrm{O}_{6}$ & Mechanical Engineering Block & $\mathrm{D}_{6}$ \\
\hline Paul Hall & $\mathrm{O}_{7}$ & Civil Engineering Block & $\mathrm{D}_{7}$ \\
\hline John Hall & $\mathrm{O}_{8}$ & Electrical and Electronics Engineering Block & $\mathrm{D}_{8}$ \\
\hline Joseph Hall & $\mathrm{O}_{9}$ & & \\
\hline Daniel Hall & $\mathrm{O}_{10}$ & & \\
\hline
\end{tabular}


Table 2. Transportation tableau.

\begin{tabular}{|c|c|c|c|c|c|c|c|c|c|}
\hline & $\mathrm{D}_{1}$ & $\mathrm{D}_{2}$ & $\mathrm{D}_{3}$ & $\mathrm{D}_{4}$ & $\mathrm{D}_{5}$ & $\mathrm{D}_{6}$ & $\mathrm{D}_{7}$ & $\mathrm{D}_{8}$ & $\begin{array}{l}\text { Actual Distance } \\
\quad(\mathrm{m} \times 100)\end{array}$ \\
\hline $\mathrm{O}_{1}$ & 10 & 8 & 11 & 11 & 11 & 16 & 17 & 20 & 9 \\
\hline $\mathrm{O}_{2}$ & 14 & 11 & 15 & 14 & 15 & 19 & 20 & 23 & 11 \\
\hline $\mathrm{O}_{3}$ & 12 & 10 & 13 & 13 & 13 & 18 & 19 & 22 & 10 \\
\hline $\mathrm{O}_{4}$ & 13 & 11 & 14 & 14 & 14 & 19 & 20 & 23 & 11 \\
\hline $\mathrm{O}_{5}$ & 13 & 11 & 14 & 14 & 14 & 19 & 20 & 23 & 10 \\
\hline $\mathrm{O}_{6}$ & 12 & 11 & 14 & 14 & 14 & 22 & 24 & 23 & 11 \\
\hline $\mathrm{O}_{7}$ & 15 & 14 & 16 & 16 & 17 & 21 & 23 & 25 & 12 \\
\hline $\mathrm{O}_{8}$ & 16 & 14 & 17 & 17 & 17 & 22 & 23 & 26 & 13 \\
\hline $\mathrm{O}_{9}$ & 15 & 13 & 16 & 16 & 16 & 21 & 22 & 25 & 11 \\
\hline $\mathrm{O}_{10}$ & 14 & 13 & 16 & 16 & 16 & 20 & 22 & 24 & 12 \\
\hline $\begin{array}{l}\text { Expected Distance } \\
\qquad(\mathrm{m} \times 100)\end{array}$ & 11 & 10 & 12 & 12 & 13 & 16 & 17 & 19 & \\
\hline
\end{tabular}

constraint quantity values which are referred to as rim requirements. In a time transportation problem, the time of transportation from $m$ origins to $n$ destinations is minimized, satisfying certain condition in respect of availability at sources and requirements at the destinations. From Table 2, we have a balanced transportation problem in which the total demand (expected distance) equals the total supply (actual distance). The distance is measured in meter $\times 100$ while the time or duration is measured in minutes.

\section{Problem Solution}

Solving transportation problems involves a lot of methods, some of them heuristic in nature [26]-[31]. In this paper, the transportation problem is solved based on the data collected, which is relatively reliable. The first stage of the solution process was to find the initial basic feasible solution [32]. The following three methods of obtaining the initial solution were used. These methods are North-west corner method, least cost method and Vogel's approximation method. Based on the Least cost method that gave the minimum time spent an optimality test was carried out, after checking for degeneracy, to determine an optimal solution using the MODI (Modified Distribution) method.

\subsection{North-West Corner Method}

Using the North West corner method to obtain an initial basic solution the values derived are shown below (Table 3 ).

\subsection{Least Cost Method}

Using the least cost method we obtain the following values as shown below (Table 4). 
Table 3. North-west corner method matrix.

Total time spent

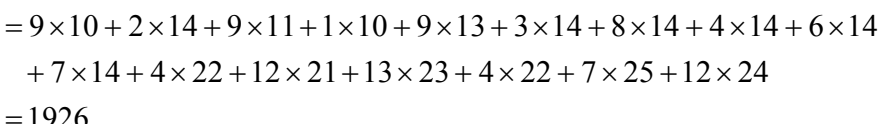

$=1926$

\begin{tabular}{cccccccccc}
\hline & $\mathrm{D}_{1}$ & $\mathrm{D}_{2}$ & $\mathrm{D}_{3}$ & $\mathrm{D}_{4}$ & $\mathrm{D}_{5}$ & $\mathrm{D}_{6}$ & $\mathrm{D}_{7}$ & $\mathrm{D}_{8}$ & $\begin{array}{c}\text { Actual Distance } \\
(\mathrm{Km})\end{array}$ \\
\hline $\mathrm{O}_{1}$ & $\mathbf{9}^{10}$ & 8 & 11 & 11 & 11 & 16 & 17 & 20 & 9 \\
$\mathrm{O}_{2}$ & $\mathbf{2}^{14}$ & $\mathbf{9}^{11}$ & 15 & 14 & 15 & 19 & 20 & 23 & 11 \\
$\mathrm{O}_{3}$ & 12 & $\mathbf{1}^{10}$ & $\mathbf{9}^{13}$ & 13 & 13 & 18 & 19 & 22 & 10 \\
$\mathrm{O}_{4}$ & 13 & 11 & $\mathbf{3}^{14}$ & $\mathbf{8}^{14}$ & 14 & 19 & 20 & 23 & 11 \\
$\mathrm{O}_{5}$ & 13 & 11 & 14 & $\mathbf{4}^{14}$ & $\mathbf{6}^{14}$ & 19 & 20 & 23 & 10 \\
$\mathrm{O}_{6}$ & 12 & 11 & 14 & 14 & $\mathbf{7}^{14}$ & $\mathbf{4}^{22}$ & 24 & 23 & 11 \\
$\mathrm{O}_{7}$ & 15 & 14 & 16 & 16 & 17 & $12^{21}$ & 23 & 25 & 12 \\
$\mathrm{O}_{8}$ & 16 & 14 & 17 & 17 & 17 & 22 & $13^{23}$ & 26 & 13 \\
$\mathrm{O}_{9}$ & 15 & 13 & 16 & 16 & 16 & 21 & $\mathbf{4}^{22}$ & $\mathbf{7}^{25}$ & 11 \\
$\mathrm{O}_{10}$ & 14 & 13 & 16 & 16 & 16 & 20 & 22 & 12 & 12 \\
Expected Distance & 11 & 10 & 12 & 12 & 13 & 16 & 17 & 19 & \\
$(\mathrm{Km})$ & & & & & & & & &
\end{tabular}

Table 4. Least cost method matrix.

Total time spent

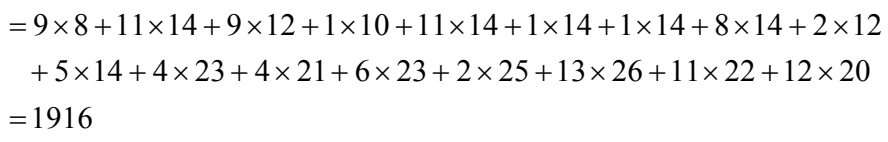

\begin{tabular}{|c|c|c|c|c|c|c|c|c|c|}
\hline & $\mathrm{D}_{1}$ & $\mathrm{D}_{2}$ & $\mathrm{D}_{3}$ & $\mathrm{D}_{4}$ & $\mathrm{D}_{5}$ & $\mathrm{D}_{6}$ & $\mathrm{D}_{7}$ & $\mathrm{D}_{8}$ & $\begin{array}{l}\text { Actual Distance } \\
(\mathrm{Km})\end{array}$ \\
\hline $\mathrm{O}_{1}$ & 10 & $9^{8}$ & 11 & 11 & 11 & 16 & 17 & 20 & 9 \\
\hline $\mathrm{O}_{2}$ & 14 & 11 & 15 & $11^{14}$ & 15 & 19 & 20 & 23 & 11 \\
\hline $\mathrm{O}_{3}$ & $9^{12}$ & $1^{10}$ & 13 & 13 & 13 & 18 & 19 & 22 & 10 \\
\hline $\mathrm{O}_{4}$ & 13 & 11 & $11^{14}$ & 14 & 14 & 19 & 20 & 23 & 11 \\
\hline $\mathrm{O}_{5}$ & 13 & 11 & $1^{14}$ & $1^{14}$ & $8^{14}$ & 19 & 20 & 23 & 10 \\
\hline $\mathrm{O}_{6}$ & $2^{12}$ & 11 & 14 & 14 & $5^{14}$ & 22 & 24 & $4^{23}$ & 11 \\
\hline $\mathrm{O}_{7}$ & 15 & 14 & 16 & 16 & 17 & $4^{21}$ & $6^{23}$ & $2^{25}$ & 12 \\
\hline $\mathrm{O}_{8}$ & 16 & 14 & 17 & 17 & 17 & 22 & 23 & $13^{26}$ & 13 \\
\hline $\mathrm{O}_{9}$ & 15 & 13 & 16 & 16 & 16 & 21 & $11^{22}$ & 25 & 11 \\
\hline $\mathrm{O}_{10}$ & 14 & 13 & 16 & 16 & 16 & $12^{20}$ & 22 & 24 & 12 \\
\hline $\begin{array}{l}\text { Expected Distance } \\
\qquad(\mathrm{Km})\end{array}$ & 11 & 10 & 12 & 12 & 13 & 16 & 17 & 19 & \\
\hline
\end{tabular}




\subsection{Test for Degeneracy}

The only optimality test condition is transportation degeneracy. Out of the three methods used to find the initial basic feasible solution to the transportation problem under consideration, the least cost method gave the minimum value of 1916. Therefore the least cost matrix is used to check for degeneracy:

$$
m+n-1=10+8-1=17
$$

The basic cells are also 17. This implies there is no problem of degeneracy thus optimality test is carried out (Table 5).

\subsection{Test for Optimality}

From the results obtained using the three methods of finding the initial basic feasible solution, least cost method gave the least amount of total time spent. Thus, its solution will be used to test for optimality in order to obtain the optimal solution (Table 6).

\subsection{Modified Distribution Method (Modi Method)}

This method involves two formulas in arriving at an optimal solution.

$U_{i}+V_{j}=C_{i j}$, which is used in determining the values of $U_{i}$ and $V_{j}$ using the values in the occupied cells.

$U_{i}-V_{j}-C_{i j}=K_{i j}$, which is used in determining the best empty route that can

Table 5. Vogel method.

$$
\begin{aligned}
& \text { Total time spent } \\
& =2 \times 11+7 \times 11+11 \times 4+10 \times 11+10 \times 12+3 \times 19+8 \times 23+6 \times 14+4 \times 20 \\
& \quad+11 \times 14+1 \times 16+11 \times 25+13 \times 22+10 \times 16+12 \times 22 \\
& =1926
\end{aligned}
$$

\begin{tabular}{cccccccccc}
\hline & $\mathrm{D}_{1}$ & $\mathrm{D}_{2}$ & $\mathrm{D}_{3}$ & $\mathrm{D}_{4}$ & $\mathrm{D}_{5}$ & $\mathrm{D}_{6}$ & $\mathrm{D}_{7}$ & $\mathrm{D}_{8}$ & $\begin{array}{c}\text { Actual Distance } \\
(\mathrm{Km})\end{array}$ \\
\hline $\mathrm{O}_{1}$ & 10 & 8 & 11 & $\mathbf{2}^{11}$ & $\mathbf{7}^{11}$ & 16 & 17 & 20 & 9 \\
$\mathrm{O}_{2}$ & $\mathbf{1 1 ^ { 4 }}$ & $\mathbf{1 0 ^ { 1 1 }}$ & 15 & 14 & 15 & 19 & 20 & 23 & 11 \\
$\mathrm{O}_{3}$ & $\mathbf{1 0 ^ { 1 2 }}$ & 10 & 13 & 13 & 13 & 18 & 19 & 22 & 10 \\
$\mathrm{O}_{4}$ & 13 & 11 & 14 & 14 & 14 & $\mathbf{3}^{19}$ & 20 & $\mathbf{8}^{23}$ & 11 \\
$\mathrm{O}_{5}$ & 13 & 11 & 14 & 14 & $\mathbf{6}^{14}$ & 19 & $\mathbf{4}^{20}$ & 23 & 10 \\
$\mathrm{O}_{6}$ & 12 & 11 & $11^{14}$ & 14 & 14 & 22 & 24 & 23 & 11 \\
$\mathrm{O}_{7}$ & 15 & 14 & 16 & 16 & 17 & 21 & $\mathbf{1}^{23}$ & $11^{25}$ & 12 \\
$\mathrm{O}_{8}$ & 16 & 14 & 17 & 17 & 17 & $13^{22}$ & 23 & 26 & 13 \\
$\mathrm{O}_{9}$ & 15 & 13 & $\mathbf{1}^{16}$ & $\mathbf{1 0}$ & 16 & 21 & 22 & 25 & 11 \\
$\mathrm{O}_{10}$ & 14 & 13 & 16 & 16 & 16 & 20 & $12^{22}$ & 24 & 12 \\
Expected Distance \\
$($ Km)
\end{tabular}


Table 6. Modified least cost method matrix.

\begin{tabular}{|c|c|c|c|c|c|c|c|c|c|c|}
\hline & & $\begin{array}{c}\mathrm{D}_{1} \\
V_{1}=\end{array}$ & $\begin{array}{c}\mathrm{D}_{2} \\
V_{2}=\end{array}$ & $\begin{array}{c}\mathrm{D}_{3} \\
V_{3}=\end{array}$ & $\begin{array}{c}\mathrm{D}_{4} \\
V_{4}=\end{array}$ & $\begin{array}{c}\mathrm{D}_{5} \\
V_{5}=\end{array}$ & $\begin{array}{c}\mathrm{D}_{6} \\
V_{6}=\end{array}$ & $\begin{array}{c}\mathrm{D}_{7} \\
V_{7}=\end{array}$ & $\begin{array}{c}\mathrm{D}_{8} \\
V_{8}=\end{array}$ & $\begin{array}{c}\text { Actual } \\
\text { Distance } \\
(\mathrm{Km})\end{array}$ \\
\hline$U_{1}=$ & $\mathrm{O}_{1}$ & 10 & $9^{8}$ & 11 & 11 & 11 & 16 & 17 & 20 & 9 \\
\hline$U_{2}=$ & $\mathrm{O}_{2}$ & 14 & 11 & 15 & $11^{14}$ & 15 & 19 & 20 & 23 & 11 \\
\hline$U_{3}=$ & $\mathrm{O}_{3}$ & $9^{12}$ & $1^{10}$ & 13 & 13 & 13 & 18 & 19 & 22 & 10 \\
\hline$U_{4}=$ & $\mathrm{O}_{4}$ & 13 & 11 & $11^{14}$ & 14 & 14 & 19 & 20 & 23 & 11 \\
\hline$U_{5}=$ & $\mathrm{O}_{5}$ & 13 & 11 & $1^{14}$ & $1^{14}$ & $8^{14}$ & 19 & 20 & 23 & 10 \\
\hline$U_{6}=$ & $\mathrm{O}_{6}$ & $2^{12}$ & 11 & 14 & 14 & $5^{14}$ & 22 & 24 & $4^{23}$ & 11 \\
\hline$U_{7}=$ & $\mathrm{O}_{7}$ & 15 & 14 & 16 & 16 & 17 & $4^{21}$ & $6^{23}$ & $2^{25}$ & 12 \\
\hline$U_{8}=$ & $\mathrm{O}_{8}$ & 16 & 14 & 17 & 17 & 17 & 22 & 23 & $13^{26}$ & 13 \\
\hline$U_{9}=$ & $\mathrm{O}_{9}$ & 15 & 13 & 16 & 16 & 16 & 21 & $11^{22}$ & 25 & 11 \\
\hline \multirow[t]{3}{*}{$U_{10}=$} & $\mathrm{O}_{10}$ & 14 & 13 & 16 & 16 & 16 & $12^{20}$ & 22 & 24 & 12 \\
\hline & Expected & & & & & & & & & \\
\hline & $\begin{array}{c}\text { Distance } \\
(\mathrm{Km})\end{array}$ & 11 & 10 & 12 & 12 & 13 & 16 & 17 & 19 & \\
\hline
\end{tabular}

be followed to reduce the total time spent and it is only the route with the most negative $K_{i j}$ that will be considered.

$U_{i}$ and $V_{j}$ are the row and column values which will be determined while $C_{i j}$ and $K_{i j}$ are the unit time and time increase or decrease respectively.

Calculating for occupied cells gives:

$$
\begin{gathered}
U_{i}+V_{j}=C_{i j} \\
\mathrm{O}_{1} \mathrm{D}_{2}: U_{1}+V_{2}=8 \\
\mathrm{O}_{2} \mathrm{D}_{4}: U_{2}+V_{4}=14 \\
\mathrm{O}_{3} \mathrm{D}_{1}: U_{3}+V_{1}=12 \\
\mathrm{O}_{3} \mathrm{D}_{2}: U_{3}+V_{2}=10 \\
\mathrm{O}_{4} \mathrm{D}_{3}: U_{4}+V_{3}=14 \\
\mathrm{O}_{5} \mathrm{D}_{3}: U_{5}+V_{3}=14 \\
\mathrm{O}_{5} \mathrm{D}_{4}: U_{5}+V_{4}=14 \\
\mathrm{O}_{5} \mathrm{D}_{5}: U_{5}+V_{5}=14 \\
\mathrm{O}_{6} \mathrm{D}_{1}: U_{6}+V_{1}=12 \\
\mathrm{O}_{6} \mathrm{D}_{5}: U_{6}+V_{5}=14 \\
\mathrm{O}_{6} \mathrm{D}_{8}: U_{6}+V_{8}=23 \\
\mathrm{O}_{7} \mathrm{D}_{6}: U_{7}+V_{6}=21 \\
\mathrm{O}_{7} \mathrm{D}_{7}: U_{7}+V_{7}=23 \\
\mathrm{O}_{7} \mathrm{D}_{8}: U_{7}+V_{8}=25 \\
\mathrm{O}_{8} \mathrm{D}_{8}: U_{8}+V_{8}=26
\end{gathered}
$$




$$
\begin{gathered}
\mathrm{O}_{9} \mathrm{D}_{7}: U_{9}+V_{7}=22 \\
\mathrm{O}_{10} \mathrm{D}_{6}: U_{10}+V_{6}=20
\end{gathered}
$$

From the equations above there are eighteen (18) unknowns but seventeen (17) equations. To solve this, 0 is assigned to one of the unknowns.

Let $U_{1}=0$

From Equation (1),

$$
\begin{gathered}
\mathrm{O}_{1} \mathrm{D}_{2}: U_{1}+V_{2}=8 \\
0+V_{2}=8
\end{gathered}
$$

Therefore, $V_{2}=8$

Substituting Equation (18) into Equation (4) gives

$$
U_{3}=2
$$

Substituting Equation (19) into Equation (3) gives

$$
V_{1}=10
$$

Substituting Equation (19) into Equation (9) gives

$$
U_{6}=2
$$

Substituting Equation (21) into Equation (10) gives

$$
V_{5}=12
$$

Substituting Equation (21) into Equation (11) gives

$$
V_{8}=21
$$

Substituting Equation (23) into Equation (14) gives

$$
U_{7}=4
$$

Substituting Equation (23) into Equation (15) gives

$$
U_{8}=5
$$

Substituting Equation (22) into Equation (8) gives

$$
U_{5}=2
$$

Substituting Equation (26) into Equation (7) gives

$$
V_{4}=12
$$

Substituting Equation (21) into Equation (2) gives

$$
U_{2}=2
$$

Substituting Equation (26) into Equation (6) gives

$$
V_{3}=12
$$

Substituting Equation (30) into Equation (5) gives

$$
U_{4}=2
$$

Substituting Equation (24) into Equation (12) gives

$$
V_{6}=17
$$

Substituting Equation (24) into Equation (13) gives 


$$
V_{7}=19
$$

Substituting Equation (33) into Equation (16) gives

$$
U_{9}=3
$$

Substituting Equation (32) into Equation (17) gives

$$
U_{10}=3
$$

Calculating for the unoccupied cells gives:

$$
\begin{gathered}
C_{i j}-U_{i}-V_{j}=K_{i j} \\
\mathrm{O}_{1} \mathrm{D}_{1}: 10-U_{1}-V_{1}=10-0-10=0 \\
\mathrm{O}_{1} \mathrm{D}_{3}: 11-U_{1}-V_{3}=11-0-12=-1 \\
\mathrm{O}_{1} \mathrm{D}_{4}: 11-U_{1}-V_{4}=11-0-12=-1 \\
\mathrm{O}_{1} \mathrm{D}_{5}: 11-U_{1}-V_{5}=11-0-12=-1 \\
\mathrm{O}_{1} \mathrm{D}_{6}: 16-U_{1}-V_{6}=16-0-17=-1 \\
\mathrm{O}_{1} \mathrm{D}_{7}: 17-U_{1}-V_{7}=17-0-19=-2 \\
\mathrm{O}_{1} \mathrm{D}_{8}: 20-U_{1}-V_{8}=20-0-21=-1 \\
\mathrm{O}_{2} \mathrm{D}_{1}: 14-U_{2}-V_{1}=14-2-10=4 \\
\mathrm{O}_{2} \mathrm{D}_{2}: 11-U_{2}-V_{2}=11-2-8=1 \\
\mathrm{O}_{2} \mathrm{D}_{3}: 15-U_{2}-V_{3}=15-2-12=-1 \\
\mathrm{O}_{2} \mathrm{D}_{5}: 15-U_{2}-V_{5}=15-2-12=-1 \\
\mathrm{O}_{2} \mathrm{D}_{6}: 19-U_{2}-V_{6}=19-2-17=0 \\
\mathrm{O}_{2} \mathrm{D}_{7}: 20-U_{2}-V_{7}=20-2-19=-1 \\
\mathrm{O}_{2} \mathrm{D}_{8}: 23-U_{2}-V_{8}=23-2-21=0 \\
\mathrm{O}_{3} \mathrm{D}_{3}: 13-U_{3}-V_{3}=13-2-12=-1 \\
\mathrm{O}_{3} \mathrm{D}_{4}: 13-U_{3}-V_{4}=13-2-12=-1 \\
\mathrm{O}_{3} \mathrm{D}_{5}: 13-U_{3}-V_{5}=13-2-12=-1 \\
\mathrm{O}_{3} \mathrm{D}_{6}: 18-U_{3}-V_{6}=18-2-17=-1 \\
\mathrm{O}_{3} \mathrm{D}_{7}: 19-U_{3}-V_{7}=19-2-19=-2 \\
\mathrm{O}_{3} \mathrm{D}_{8}: 22-U_{3}-V_{8}=22-2-21=-1 \\
\mathrm{O}_{4} \mathrm{D}_{1}: 13-U_{4}-V_{1}=13-12-10=-9 \\
\mathrm{O}_{4} \mathrm{D}_{2}: 11-U_{4}-V_{2}=11-12-8=-9 \\
\mathrm{O}_{4} \mathrm{D}_{4}: 14-U_{4}-V_{4}=14-12-12=-10 \\
\mathrm{O}_{4} \mathrm{D}_{5}: 14-U_{4}-V_{5}=14-12-12=-10 \\
\mathrm{O}_{4} \mathrm{D}_{6}: 19-U_{4}-V_{6}=19-12-17=-10 \\
\mathrm{O}_{4} \mathrm{D}_{7}: 20-U_{4}-V_{7}=20-12-19=-11 \\
\mathrm{O}_{4}-V_{8}=23-21-10=-10 \\
\mathrm{O}_{5}: 2-10=1
\end{gathered}
$$




$$
\begin{aligned}
& \mathrm{O}_{5} \mathrm{D}_{2}: 11-U_{5}-V_{2}=11-2-8=1 \\
& \mathrm{O}_{5} \mathrm{D}_{6}: 19-U_{5}-V_{6}=19-2-17=0 \\
& \mathrm{O}_{5} \mathrm{D}_{7}: 20-U_{5}-V_{7}=20-2-19=-1 \\
& \mathrm{O}_{5} \mathrm{D}_{8}: 23-U_{5}-V_{8}=23-2-21=0 \\
& \mathrm{O}_{6} \mathrm{D}_{2}: 11-U_{6}-V_{2}=11-2-8=1 \\
& \mathrm{O}_{6} \mathrm{D}_{3}: 14-U_{6}-V_{3}=14-2-12=0 \\
& \mathrm{O}_{6} \mathrm{D}_{4}: 14-U_{6}-V_{4}=14-2-12=0 \\
& \mathrm{O}_{6} \mathrm{D}_{6}: 22-U_{6}-V_{6}=22-2-17=3 \\
& \mathrm{O}_{6} \mathrm{D}_{7}: 24-U_{6}-V_{7}=24-2-19=3 \\
& \mathrm{O}_{7} \mathrm{D}_{1}: 15-U_{7}-V_{1}=15-4-10=1 \\
& \mathrm{O}_{7} \mathrm{D}_{2}: 14-U_{7}-V_{2}=14-4-8=2 \\
& \mathrm{O}_{7} \mathrm{D}_{3}: 16-U_{7}-V_{3}=16-4-12=0 \\
& \mathrm{O}_{7} \mathrm{D}_{4}: 16-U_{7}-V_{4}=16-4-12=0 \\
& \mathrm{O}_{7} \mathrm{D}_{5}: 17-U_{7}-V_{5}=17-4-12=1 \\
& \mathrm{O}_{8} \mathrm{D}_{1}: 16-U_{8}-V_{1}=16-5-10=1 \\
& \mathrm{O}_{8} \mathrm{D}_{2}: 14-U_{8}-V_{2}=14-5-8=1 \\
& \mathrm{O}_{8} \mathrm{D}_{3}: 17-U_{8}-V_{3}=17-5-12=0 \\
& \mathrm{O}_{8} \mathrm{D}_{4}: 17-U_{8}-V_{4}=17-5-12=0 \\
& \mathrm{O}_{8} \mathrm{D}_{5}: 17-U_{8}-V_{5}=17-5-12=0 \\
& \mathrm{O}_{8} \mathrm{D}_{6}: 22-U_{8}-V_{6}=22-5-17=0 \\
& \mathrm{O}_{8} \mathrm{D}_{7}: 23-U_{8}-V_{7}=23-5-19=-1 \\
& \mathrm{O}_{9} \mathrm{D}_{1}: 15-U_{9}-V_{1}=15-3-10=2 \\
& \mathrm{O}_{9} \mathrm{D}_{2}: 13-U_{9}-V_{2}=13-3-8=2 \\
& \mathrm{O}_{9} \mathrm{D}_{3}: 16-U_{9}-V_{3}=16-3-12=1 \\
& \mathrm{O}_{9} \mathrm{D}_{4}: 16-U_{9}-V_{4}=16-3-12=1 \\
& \mathrm{O}_{9} \mathrm{D}_{5}: 16-U_{9}-V_{5}=16-3-12=1 \\
& \mathrm{O}_{9} \mathrm{D}_{6}: 21-U_{9}-V_{6}=21-3-17=1 \\
& \mathrm{O}_{9} \mathrm{D}_{8}: 25-U_{9}-V_{8}=25-3-21=2 \\
& \mathrm{O}_{10} \mathrm{D}_{1}: 14-U_{10}-V_{1}=14-3-10=1 \\
& \mathrm{O}_{10} \mathrm{D}_{2}: 13-U_{10}-V_{2}=13-3-8=2 \\
& \mathrm{O}_{10} \mathrm{D}_{3}: 16-U_{10}-V_{3}=16-3-12=1 \\
& \mathrm{O}_{10} \mathrm{D}_{4}: 16-U_{10}-V_{4}=16-3-12=1 \\
& \mathrm{O}_{10} \mathrm{D}_{5}: 16-U_{10}-V_{5}=16-3-12=1 \\
& \mathrm{O}_{10} \mathrm{D}_{7}: 16-U_{10}-V_{7}=16-3-19=-6 \\
& \mathrm{O}_{10} \mathrm{D}_{8}: 24-U_{10}-V_{8}=24-3-21=0
\end{aligned}
$$


Table 7. MODI method iteration showing the new allocation.

Total time

$$
\begin{aligned}
= & 9 \times 8+11 \times 14+9 \times 12+1 \times 10+5 \times 14+6 \times 20+1 \times 14+1 \times 14+8 \times 14+2 \times 12 \\
& +5 \times 14+4 \times 23+6 \times 16+4 \times 21+2 \times 25+13 \times 26+11 \times 22+12 \times 20 \\
= & 1896
\end{aligned}
$$

\begin{tabular}{|c|c|c|c|c|c|c|c|c|c|c|}
\hline & & $\begin{array}{c}\mathrm{D}_{1} \\
V_{1}=\end{array}$ & $\begin{array}{c}\mathrm{D}_{2} \\
V_{2}=\end{array}$ & $\begin{array}{c}\mathrm{D}_{3} \\
V_{3}=\end{array}$ & $\begin{array}{c}\mathrm{D}_{4} \\
V_{4}=\end{array}$ & $\begin{array}{c}\mathrm{D}_{5} \\
V_{5}=\end{array}$ & $\begin{array}{c}\mathrm{D}_{6} \\
V_{6}=\end{array}$ & $\begin{array}{c}\mathrm{D}_{7} \\
V_{7}=\end{array}$ & $\begin{array}{c}\mathrm{D}_{8} \\
V_{8}=\end{array}$ & $\begin{array}{c}\text { Actual } \\
\text { Distance } \\
(\mathrm{Km})\end{array}$ \\
\hline$U_{1}=$ & $\mathrm{O}_{1}$ & 10 & $9^{8}$ & 11 & 11 & 11 & 16 & 17 & 20 & 9 \\
\hline$U_{2}=$ & $\mathrm{O}_{2}$ & 14 & 11 & 15 & $11^{14}$ & 15 & 19 & 20 & 23 & 11 \\
\hline$U_{3}=$ & $\mathrm{O}_{3}$ & $9^{12}$ & $1^{10}$ & 13 & 13 & 13 & 18 & 19 & 22 & 10 \\
\hline$U_{4}=$ & $\mathrm{O}_{4}$ & 13 & 11 & $5(-)^{14}$ & 14 & 14 & 19 & $6(+)^{20}$ & 23 & 11 \\
\hline$U_{5}=$ & $\mathrm{O}_{5}$ & 13 & 11 & $1^{14}$ & $1^{14}$ & $8^{14}$ & 19 & 20 & 23 & 10 \\
\hline$U_{6}=$ & $\mathrm{O}_{6}$ & $2^{12}$ & 11 & 14 & 14 & $5^{14}$ & 22 & 24 & $4^{23}$ & 11 \\
\hline$U_{7}=$ & $\mathrm{O}_{7}$ & 15 & 14 & $6(+)^{16}$ & 16 & 17 & $4^{21}$ & $(-)^{23}$ & $2^{25}$ & 12 \\
\hline$U_{8}=$ & $\mathrm{O}_{8}$ & 16 & 14 & 17 & 17 & 17 & 22 & 23 & $13^{26}$ & 13 \\
\hline$U_{9}=$ & $\mathrm{O}_{9}$ & 15 & 13 & 16 & 16 & 16 & 21 & $11^{22}$ & 25 & 11 \\
\hline \multirow[t]{3}{*}{$U_{10}=$} & $\mathrm{O}_{10}$ & 14 & 13 & 16 & 16 & 16 & $12^{20}$ & 22 & 24 & 12 \\
\hline & Expected & & & & & & & & & \\
\hline & $\begin{array}{c}\text { Distance } \\
(\mathrm{Km})\end{array}$ & 11 & 10 & 12 & 12 & 13 & 16 & 17 & 19 & \\
\hline
\end{tabular}

From the calculations above, $\mathrm{O}_{4} \mathrm{D}_{7}$ is the only cell with the most negative $K_{i j}$ with -11 . With this result it means that the total time will be reduced by -11 if we allocate to that cell. The Table 7 illustrates this.

\section{Result Discussion}

From the analysis carried out with the data collected, it can be seen that least cost method gave the best initial basic feasible solution. Optimality test carried out, using MODI method, gave an optimal value of 1896 . This was an improvement on the result, 1916, given by the least cost method. Also, it can be seen that by allocating maximally to route $\mathrm{O}_{4} \mathrm{D}_{7}$, the total time will be reduced by -11 . From Table 7 above, it can be seen that the total time can be minimized if $900 \mathrm{~m}$ is covered using route $\mathrm{O}_{1} \mathrm{D}_{2}, 11 \mathrm{~km}$ covered using $\mathrm{O}_{2} \mathrm{D}_{4}, 9 \mathrm{~km}$ covered using $\mathrm{O}_{3} \mathrm{D}_{1}, 1 \mathrm{~km}$ covered using $\mathrm{O}_{3} \mathrm{D}_{2}, 5 \mathrm{~km}$ covered using $\mathrm{O}_{4} \mathrm{D}_{3}, 6 \mathrm{~km}$ covered using $\mathrm{O}_{4} \mathrm{D} 7,1 \mathrm{~km}$ covered using $\mathrm{O}_{5} \mathrm{D}_{3}, 1 \mathrm{~km}$ covered using $\mathrm{O}_{5} \mathrm{D}_{4}, 8 \mathrm{~km}$ covered using $\mathrm{O}_{5} \mathrm{D}_{5}, 2 \mathrm{~km}$ covered using $\mathrm{O}_{6} \mathrm{D}_{1}, 5 \mathrm{~km}$ covered using $\mathrm{O}_{6} \mathrm{D}_{5}, 4 \mathrm{~km}$ covered using $\mathrm{O}_{6} \mathrm{D}_{8}, 6 \mathrm{~km}$ covered using $\mathrm{O}_{7} \mathrm{D}_{3}, 400 \mathrm{~m}$ covered using $\mathrm{O}_{7} \mathrm{D}_{6}, 13 \mathrm{~km}$ covered using $\mathrm{O}_{8} \mathrm{D}_{8}, 11 \mathrm{~km}$ covered using $\mathrm{O}_{9} \mathrm{D}_{7}, 1200 \mathrm{~m}$ covered using $\mathrm{O}_{10} \mathrm{D}_{6}$. Also, from the table it can be seen that some requires less time to cover than the others. For instance, route $\mathrm{O}_{1} \mathrm{D}_{2}$ requires just 8 minutes to cover $900 \mathrm{~m}$ while some other routes, like route $\mathrm{O}_{5} \mathrm{D}_{3}$ and route $\mathrm{O}_{5} \mathrm{D}_{4}$, require a lot of time. This could be as a result of different factors relating to the transportation system generally which 
the university authority should look into. Also, this analysis and the result would help the university to plan in order to optimize its transportation system within the campus, especially as regards the movement of students to and fro their lecture rooms.

\section{Conclusion}

In this paper time minimizing transportation model was used to determine how the movement of students by road from their hostels to their different lecture rooms can be optimized. Three methods were used to arrive at an initial basic feasible solution and Least Cost method was found to give the least total time as compared to the other two methods. MODI method was used to obtain an optimal solution. From the result derived, it shows that the total time spent on the movement of students to their lecture rooms, from their different hostels, can be minimized thereby improving campus life and reducing lateness to lectures. Covenant University can use this to plan and manage the transportation system of the university, as it thrives on becoming one of the best universities in the world.

\section{References}

[1] Mitchell, R.B. (1962) Transportation Problems and Their Solutions. Symposium on Metropolitan Planning, 106.

[2] Agarana, M.C., Owoloko, E.A. and Kolawole, A.A. (2016) Enhancing the Movement of People and Goods in a Potential World Class University Using Transportation Model. Global Journal of Pure and Applied Mathematics, 12, 281-294.

[3] Coulson, J., et al. (2011) University Planning and Architecture: The Search for perfection. Routledge, New York.

[4] Al-Mosaind, M. (2014) Traffic Conditions in Emerging University Campuses: King Saud University, Riyadh, Saudi Arabia. Journal of Sustainable Development, 7.

[5] Michael, A. and Foluso, A. (2015) Maximizing Operations Processes of a Potential World Class University Using Mathematical Model. American Journal of Applied Mathematics, 3, 59-63. https://doi.org/10.11648/j.ajam.20150302.15

[6] Sharma, G., Abbas, S. and Gupta, V. (2015) Solving Time Minimizing Transportation Problem by Zero Point Method. International Journal of Engineering and Science, 5, 23-26.

[7] Sheng, Y. and Yao, K. (2012) Fixed Charge Transportation Problem and Its Uncertain Programming Model. Industrial Engineering and Management System, 11, 183-187. https://doi.org/10.7232/iems.2012.11.2.183

[8] Xie, F. and Jia, R. (2009) A Heuristic Algorithm for Solving Fixed-Charge Transportation Problem. 2009 WRI World Congress on Computer Science and Information Engineering, Los Angeles, 31 March-2 April 2009, 574-580. https://doi.org/10.1109/CSIE.2009.110

[9] Xie, F. and Jia, R. (2010) A Note on "Nonlinear Fixed Charge Transportation Problem by Spanning Tree-Based Genetic Algorithm" by Jung-Bok Jo, Yinzhen Li, Mitsuo Gen, Computers \& Industrial Engineering (2007). Computers \& Industrial Engineering, 59, 1013-1014. https://doi.org/10.1016/j.cie.2010.07.009

[10] Agarana, M.C. and Ehigbochie, A.I. (2015) Optimization of Student's Academic Performance in a World-Class University Using Operational Research Technique. 
International Journal of Mathematics and Computer Applications Research, 5, 43-50.

[11] Norton, R., et al. (2007) Transforming the University Campus into a Sustainable Community. Planning for Higher Education, 35, 22-39.

[12] HDR Engineering, Inc. (2012) 2012 Campus Mobility Plan: Final Report. Prepared for the North Carolina State Transportation. HDR Engineering, Inc., Omaha.

[13] Xie, F., Jia, Y. and Jia, R. (2012) Duration \& Cost Optimization for Transportation Problem.

[14] Taha, H.A. (2006) Operation Research: An Introduction. 7th Edition, Prentice Hall, Upper Saddle River.

[15] Adlakha, V. and Kowalski, K. (1999) On the Fixed-Charge Transportation Problem. Omega, 27, 381-388. https://doi.org/10.1016/S0305-0483(98)00064-4

[16] Kowalski, K. and Lev, B. (2008) On Step Fixed-Charge Transportation Problem. Omega, 36, 913-917.

[17] Gray, P. (1971) Exact Solution of the Fixed-Charge Transportation Problem. Operations Research, 19, 1529-1538. https://doi.org/10.1287/opre.19.6.1529

[18] Liu, S.-T. (2003) The Total Cost Bounds of the Transportation Problem with Varying Demand and Supply. Omega, 31, 247-251.

https://doi.org/10.1016/S0305-0483(03)00054-9

[19] Brenner, U. (2008) A Faster Polynomial Algorithm for the Unbalanced Hitchcock Transportation Problem. Operations Research Letters, 36, 408-413. https://doi.org/10.1016/j.orl.2008.01.011

[20] Russell, R.S. and Taylor, B.W. (1998) Operations Management: Focusing on Quality and Competitiveness. 2nd Edition, Prentice Hall, Inc., Upper Saddle River.

[21] Sun, M., Aronson, J.E., McKeown, P.G. and Drinka, D. (1998) A Tabu Search Heuristic Procedure for the Fixed Charge Transportation Problem. European Journal of Operational Research, 106, 441-456. https://doi.org/10.1016/S0377-2217(97)00284-1

[22] Dahiya, K. and Verma, V. (2007) Capacitated Transportation Problem with Bounds on RIM Conditions. European Journal of Operational Research, 178, 718-737. https://doi.org/10.1016/j.ejor.2006.02.017

[23] Xie, F. and Jia, R. (2009) On Capacitated Transportation Problem with Bounds on Rim Conditions. International Conference on New Trends in Information and Service Science, Beijing, 30 June-2 July 2009, 131-138. https://doi.org/10.1109/NISS.2009.17

[24] UN Habitat (2013) Planning and Design for Sustainable Urban Mobility: Global Report on Human Settlement 2013. UN Habitat, Washington DC.

[25] Adlakha, V. and Kowalski, K. (1998) A Quick Sufficient Solution to the More-for-Less Paradox in the Transportation Problems. Omega, 26, 541-547. https://doi.org/10.1016/S0305-0483(97)00070-4

[26] Agarana, M.C., Bishop, S.A. and Odetunmibi, O.A. (2014) Optimization of Banks Loan Portfolio Management Using Goal Programming Technique. International Journal of Research in Applied, Natural, Social Sciences, 2, 43-52.

[27] Agarana, M.C. (2014) An Application of Goal Programming Technique to Loan Portfolio Management in Banking Industry. In: ICAPTA 2014 Conference, University of Lagos, Mathematics Analysis and Optimization Research Group, Lagos.

[28] Adlakha, V. and Kowalski, K. (2000) A Note on the Procedure MFL for a More-for-Less Solution in Transportation Problems. Omega, 28, 481-483. 
https://doi.org/10.1016/S0305-0483(99)00074-2

[29] Adlakha, V. and Kowalski, K. (2001) A Heuristic Method for More-for-Less in Distribution Related Problems. International Journal of Mathematical Education in Science and Technology, 26, 61-71. https://doi.org/10.1080/00207390117225

[30] Adlakha, V., Kowalski, K., Vemuganti, R.R. and Lev, B. (2007) More-for-Less Algorithm for Fixed-Charge Transportation Problems. Omega, 35, 116-127. https://doi.org/10.1016/j.omega.2006.03.001

[31] Adlakha, V. and Kowalski, K. (2003) A Simple Heuristic for Solving Small Fixed-Charge Transportation Problems. Omega, 31, 205-211. https://doi.org/10.1016/S0305-0483(03)00025-2

[32] Agarana, M.C. and Olokunde, T.O. (2015) Optimization of Healthcare Pathways in Covenant University Health Centre Using Linear Programing Model. Journal of Applied Mathematics, 91, 215-228.

https://doi.org/10.17654/FJAMJun2015_215_228 